УДК 541.138.3

\title{
КИНЕТИКА РЕАКЦИИ ВЫДЕЛЕНИЯ ВОДОРОДА НА МОНОСИЛИЦИДЕ НИКЕЛЯ В КИСЛОМ И ЩЕЛОЧНОМ РАСТВОРАХ
}

\author{
() 2017 В. И. Кичигин, А. Б. Шеин, А. Ш. Шамсутдинов \\ Пермский государственный национальный исследовательский университет, \\ ул. Букирева, 15, 614990 Пермь, Россия \\ e-mail: ashein@psu.ru,kichigin@psu.ru
}

Поступила в редакцию 22.02.2017 г.

\begin{abstract}
Аннотация. Методами поляризационных измерений и импедансной спектроскопии изучена реакция выделения водорода на $\mathrm{NiSi}$-электроде в растворах $0.5 \mathrm{M} \mathrm{H}_{2} \mathrm{SO}_{4}$ и $1 \mathrm{M} \mathrm{KOH}$. Результаты электрохимических измерений позволяют предположить, что выделение водорода на $\mathrm{NiSi}$ в сернокислом растворе протекает по двухмаршрутному механизму Фольмера-Гейровского-Тафеля, а в щелочном растворе - по одномаршрутному механизму Фольмера-Гейровского.
\end{abstract}

Ключевые слова: моносилицид никеля, реакция выделения водорода, абсорбция водорода, импеданс.

\section{ВВЕДЕНИЕ}

Исследования закономерностей реакции выделения водорода (РВВ) проводятся в течение многих десятилетий, но не утратили своей актуальности [1]. Важность изучения РВВ обусловлена, среди прочего, ярко выраженной зависимостью кинетики реакции от природы материала электрода. В связи с этим в качестве катализаторов РВВ исследовались металлы, сплавы, интерметаллические соединения, композиционные материалы, различные соединения металлов (карбиды, силициды, нитриды, фосфиды, оксиды, халькогениды и др.) [2, 3].

Кинетика РВВ на силицидах переходных металлов была предметом изучения в ряде работ [4-11]. В некоторых случаях отмечались более низкие перенапряжения выделения водорода на силицидах по сравнению с соответствующими чистыми металлами $[4,5,10]$.

Для моносилицида никеля $\mathrm{NiSi}$ в $1 \mathrm{M} \mathrm{HClO}_{4}$ получены поляризационные кривые с тафелевским наклоном $b=0.113 \mathrm{~B}$, а в растворе $\mathrm{NaClO}_{4}+\mathrm{NaOH}$ $(\mathrm{pH} 10.8) b=0.145 \mathrm{~B}$; в обоих растворах плотность тока обмена $i_{\mathrm{o}}=4 \cdot 10^{-6} \mathrm{~A} / \mathrm{cm}^{2}$ [4]. Предполагается [4], что в кислом растворе лимитирующей стадией на NiSi является стадия разряда ионов водорода. Относительно механизма PBB на NiSi в щелочном растворе определенных выводов в [4] не было сделано.
Кинетика РВВ на моносилициде никеля остается недостаточно изученной. Цель данной работы изучить кинетику и механизм PBB на NiSi-электроде в кислом и щелочном растворах с использованием метода спектроскопии электрохимического импеданса.

\section{МЕТОДИКА ЭКСПЕРИМЕНТА}

Исследуемые электроды были изготовлены из силицида никеля NiSi, полученного методом Чохральского из кремния КПЗ-1 (99.99 мас.\% Si), и никеля марки Н-0 (99.99 мас.\% Ni). Рабочая площадь поверхности электродов составляла примерно $0.3 \mathrm{~cm}^{2}$. Поверхность электродов обрабатывали на тонкой шлифовальной бумаге, очищали этиловым спиртом, промывали рабочим раствором.

В качестве рабочих растворов использовались $0.5 \mathrm{M} \mathrm{H}_{2} \mathrm{SO}_{4}$ и $1 \mathrm{M} \mathrm{KOH.} \mathrm{Растворы} \mathrm{готовили} \mathrm{из}$ $\mathrm{H}_{2} \mathrm{SO}_{4}$ марки «х.ч.» или $\mathrm{KOH}$ марки «ос.ч.» и деионизованной воды (Milli-Q, удельное сопротивление 18.2 МОм·см, содержание органического углерода 3-4 мкг/л). Растворы деаэрировали водородом (чистота 99.999 \% в расчете на сухой газ), полученным электролитически в генераторе водорода «Кулон-16». Температура растворов $21-23^{\circ} \mathrm{C}$.

Измерения импеданса проводились в диапазоне частот $f$ от 10 кГц до 0.01 Гц (10 точек на декаду) в потенциостатическом режиме поляризации с 
помощью установки Solartron 1255/1287 (Solartron Analytical). Амплитуда переменного сигнала 10 мB. Потенциал электрода изменяли от более низких катодных поляризаций к более высоким. Значения потенциалов в статье отнесены к стандартному водородному электроду.

При измерениях и обработке импедансных данных использовали программы CorrWare2, ZPlot2, ZView2 (Scribner Associates, Inc.).

\section{РЕЗУЛЬТАТЫ И ИХ ОБСУЖДЕНИЕ}

\section{Раствор серной кислоты}

На рис. 1 приведена исправленная на омическое падение потенциала поляризационная кривая $\mathrm{NiSi}$-электрода в растворе серной кислоты. Плотность тока $i$ рассчитана на видимую поверхность электрода. Среднеквадратичная погрешность составляла $12 \mathrm{mB}$ (при $i=10^{-3} \mathrm{~A} / \mathrm{cm}^{2}$ ). На поляризационной кривой можно выделить два тафелевских участка: до потенциала $E \approx-0.33$ В тафелевский наклон $b$ равен $0.105 \mathrm{~B}$, при $E<-0.33 \mathrm{~B}-b=0.18 \mathrm{~B}$. Экстраполяцией поляризационной кривой до равновесного потенциала водородного электрода получена плотность тока обмена $i_{0}=4.45 \cdot 10^{-6} \mathrm{~A} / \mathrm{cm}^{2}$. Это значение $i_{0}$ и тафелевский наклон $0.105 \mathrm{~B}$ (при $E>-0.33 \mathrm{~B})$ хорошо согласуются с соответствующими величинами $\left(4 \cdot 10^{-6} \mathrm{~A} / \mathrm{cm}^{2}, 0.113 \mathrm{~B}\right)$, полученными в [4] для $\mathrm{NiSi}$ в $1 \mathrm{M} \mathrm{HClO}_{4}$.

Перенапряжение выделения водорода на $\mathrm{NiSi}$ электроде в сернокислом растворе значительно ниже, чем на $\mathrm{FeSi}$-электроде; плотность тока $1 \mathrm{~mA} / \mathrm{cm}^{2}$ на $\mathrm{NiSi}$ в $0.5 \mathrm{M} \mathrm{H}_{2} \mathrm{SO}_{4}$ достигается при

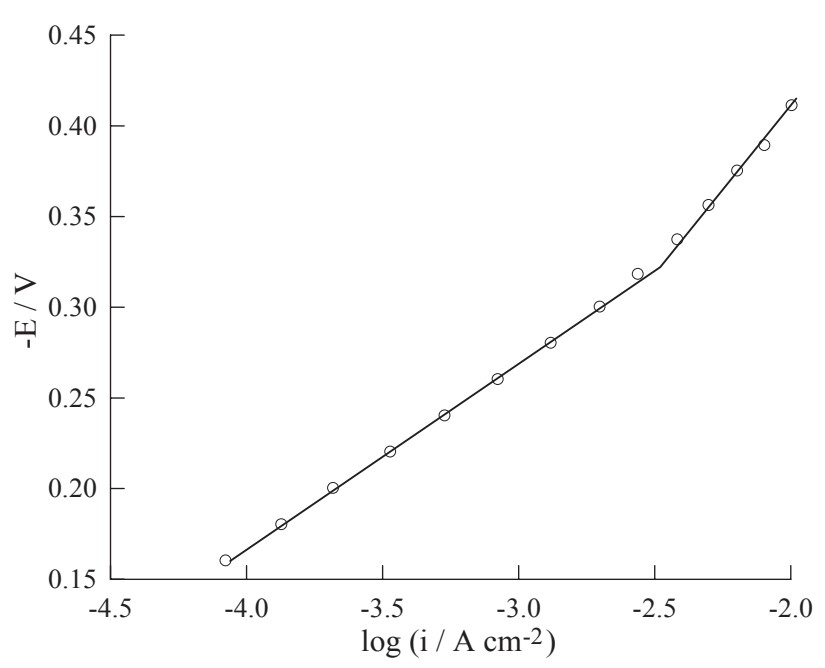

Рис. 1. Поляризационная кривая NiSi-электрода в $0.5 \mathrm{M} \mathrm{H}_{2} \mathrm{SO}_{4}$

[Fig. 1. Polarization curve for NiSi electrode in $0.5 \mathrm{M}$ $\left.\mathrm{H}_{2} \mathrm{SO}_{4}\right]$
$E=-0.27 \mathrm{~B}$, а на $\mathrm{FeSi}-$ при $E \approx-0.48 \mathrm{~B}$ [11]. В целом, форма поляризационной кривой для $\mathrm{NiSi}$ и $\mathrm{FeSi}$ различается, однако, если для FeSi рассматривать только область $i>10^{-4} \mathrm{~A} / \mathrm{cm}^{2}$ (в которой имеются данные для $\mathrm{NiSi}$ (рис. 1)), то можно отметить аналогию поляризационных кривых для обоих моносилицидов.

По соотношению $C=-1 /\left(\omega Z^{\prime \prime}\right)$, где $\omega=2 \pi f, Z^{\prime \prime}-$ мнимая составляющая импеданса при данной частоте $f$, определили емкость $C$. Кривая дифференциальной емкости $\mathrm{NiSi}$-электрода в сернокислом растворе в катодной области при достаточно высокой частоте переменного тока, когда значения емкости близки к емкости двойного электрического слоя, приведена на рис. 2. Значения удельного сопротивления $\mathrm{NiSi}$-пленок ( $\leq 40$ мкОм·см при комнатной температуре), температурная зависимость электропроводности и коэффициент Холла указывают на металлический характер моносилицида никеля [12], то есть для NiSi-электрода можно было бы ожидать величин двойнослойной емкости, характерных для твердых металлических электродов (20-30 мкФ/см²). Наблюдаемые значения дифференциальной емкости меньше указанных, но существенно превышают емкость FeSi-электрода в тех же условиях [11]. Оксидной пленки на $\mathrm{NiSi-электроде} \mathrm{в} \mathrm{исследуемой} \mathrm{области} \mathrm{потенциа-}$ лов, по-видимому, нет, однако на поверхностных атомах Si может присутствовать хемосорбированный кислород. Такой характер распределения адсорбированного кислорода на поверхности силицидов никеля был установлен при исследовании

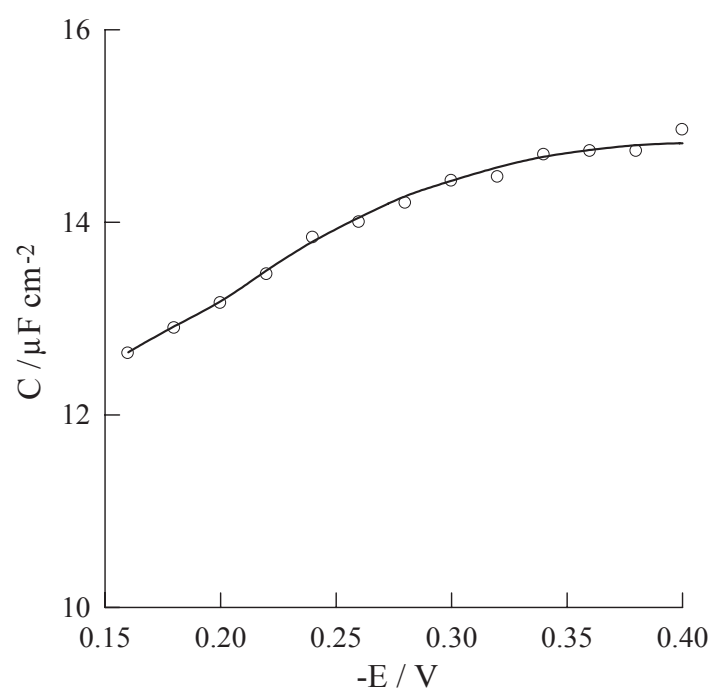

Рис. 2. Кривая дифференциальной емкости при $f=10$ кГц для NiSi-электрода в $0.5 \mathrm{M} \mathrm{H}_{2} \mathrm{SO}_{4}$

[Fig. 2. Differential capacitance at $f=10 \mathrm{kHz}$ for NiSi electrode in $0.5 \mathrm{M} \mathrm{H}_{2} \mathrm{SO}_{4}$ ] 
взаимодействия $\mathrm{Ni}_{x} \mathrm{Si}(x=0.5-3)$ с кислородом в газовой фазе при комнатной температуре [13]. Было показано, что кислород образует связи с $\mathrm{Si}$, а не с металлом; более того, в присутствии Ni окисление $\mathrm{Si}$ усиливается по сравнению с чистым Si [13]. B водных растворах поверхностные атомы $\mathrm{Si}$, вероятно, могут быть окислены за счет кислорода молекул воды. Вклад $\mathrm{Si}$-центров с адсорбированным кислородом в измеряемую емкость понижен, а поскольку в силициде количество поверхностных атомов никеля меньше, чем в чистом металле, это приводит к более низким значениям емкости двойного слоя на $\mathrm{NiSi}$ по сравнению с никелем.

Спектры импеданса при некоторых потенциалах электрода представлены на рис. 3. Здесь $Z^{\prime}$ и $Z^{\prime \prime}$ - действительная и мнимая составляющие импеданса, $\varphi$ - фазовый угол импеданса. При невысоких катодных поляризациях график Найквиста имеет вид несколько деформированной полуокружности, соответствующие графики Боде представляют собой один несимметричный пик. При достаточно высоких поляризациях появляется низкочастотная дуга. Первые отклонения в области низких частот обнаруживаются при $E=-0.28 \mathrm{~B}$, то есть при несколько более высоком потенциале по сравнению с потенциалом перехода от первого тафелевского участка ко второму. Таким образом, имеется определенное сходство импедансных характеристик FeSi- [11] и NiSi-электродов в области потенциалов выделения водорода в $0.5 \mathrm{M} \mathrm{H}_{2} \mathrm{SO}_{4}$ : при повышении катодной поляризации происходит усложнение графиков импеданса - наблюдается переход от одной емкостной дуги к двум хорошо разделенным дугам.

Для описания спектров импеданса NiSi-электрода применимы те же эквивалентные схемы (рис. 4), которые использовались в случае $\mathrm{FeSi}$ электрода [11]. Эквивалентная схема А (рис. 4) хорошо известная модель для электрода, на поверхности которого протекает РВВ с одним адсорбированным интермедиатом $[14,15]$. В эквивалентной схеме $\mathrm{A}: R_{1}, R_{2}, C_{2}$ - параметры фарадеевского импеданса, их физический смысл обсуждался в $[14,15]$; СРЕ - элемент постоянной фазы, моделирующий двойной электрический слой на неоднородной поверхности твердого электрода [16]. Такой же вид (рис. $4, \mathrm{~A}$ ) имеет эквивалентная схема для процесса $\mathrm{PBB}+$ реакция абсорбции водорода (РАВ), если абсорбция водорода происходит через состояние $\mathrm{H}_{\text {адс }}$ с кинетическим контролем [17]. Эквивалентная схема В (рис. 4) - модель границы раздела электрод/раствор в случае, когда РВВ со- провождается реакцией абсорбции водорода [15, 17], протекающей со смешанным диффузионнокинетическим контролем; здесь $R_{a b s}$ - сопротивление абсорбции, то есть стадии перехода адсорбированных атомов водорода $\mathrm{H}_{\text {адс }}$ в подповерхностное положение, $Z_{d}$ - импеданс диффузии водорода в твердой фазе: $Z_{d}=R_{d}$ th $\left(j \omega \tau_{d}\right)^{1 / 2} /\left(j \omega \tau_{d}\right)^{1 / 2}$, где $R_{d}-$ диффузионное сопротивление, $\tau_{d}-$ диффузионная временная константа.

Не удается описать экспериментальные импедансные данные во всем изученном интервале потенциалов с помощью какой-либо одной из двух эквивалентных схем - использование схемы А при $E<-0.26$ В приводит к очень высоким значениям емкости $C_{2}$, которые совершенно не согласуются с величинами $C_{2}$, полученными для схемы А при более высоких $E$, а использование схемы В при $E>-0.24$ В дает весьма большие ошибки в параметрах импеданса РАВ. Поэтому в разных интервалах $E$ использовали разные эквивалентные схемы. На рис. 5, на котором приведены зависимости параметров импеданса РВВ от потенциала электрода, области, в которых использовалась схема А или схема $\mathrm{B}$, разделены вертикальной штриховой линией. Значения $R_{1}, R_{2}$ и других элементов эквивалентных схем находили с помощью комплексного нелинейного метода наименьших квадратов (программа ZView, алгоритм Левенберга-Марквардта). В областях своей применимости эквивалентные схемы А и В хорошо описывают спектры импеданса $\mathrm{NiSi}$-электрода: статистика $\chi^{2}$ составляет $\sim 1 \cdot 10^{-4}$ для схемы А при $E>-0.24$ В и $(2-5) \cdot 10^{-5}$ для схемы В при $E \leq-0.24$ В (при использовании весовых коэффициентов, равных обратной величине модуля импеданса при соответствующей частоте). Относительные ошибки определения элементов эквивалентных схем в зависимости от $E$ составляли $1.5-6 \%$ для $R_{1}, 4.5-7 \%$ для $R_{2}$ и $5-13.5 \%$ для $C_{2}$.

Зависимость $\lg R_{1}$ от $E$ представлена одним линейным участком, зависимость $\lg R_{2}$ от $E$ состоит из двух линейных участков, причем при $E<-0.30 \mathrm{~B}$ сопротивления $R_{1}$ и $R_{2}$ близки друг к другу и $\lg R_{1}, E$ и $\lg R_{2}, E$-прямые практически параллельны (рис. 5). Емкость $C_{2}$ возрастает при понижении потенциала электрода, а при приближении к $E=-0.4$ В начинает уменьшаться (рис. 5).

Дополнительно охарактеризовать импеданс РВВ можно с помощью временной константы $\tau=R_{2} C_{2}$ для адсорбированного водорода. На рис. 6 приведена зависимость $\lg \tau$ от $E$. В интервале потенциалов от -0.22 до -0.34 В эта зависимость является прямолинейной с наклоном $3.8 \mathrm{~B}^{-1}$, а при 

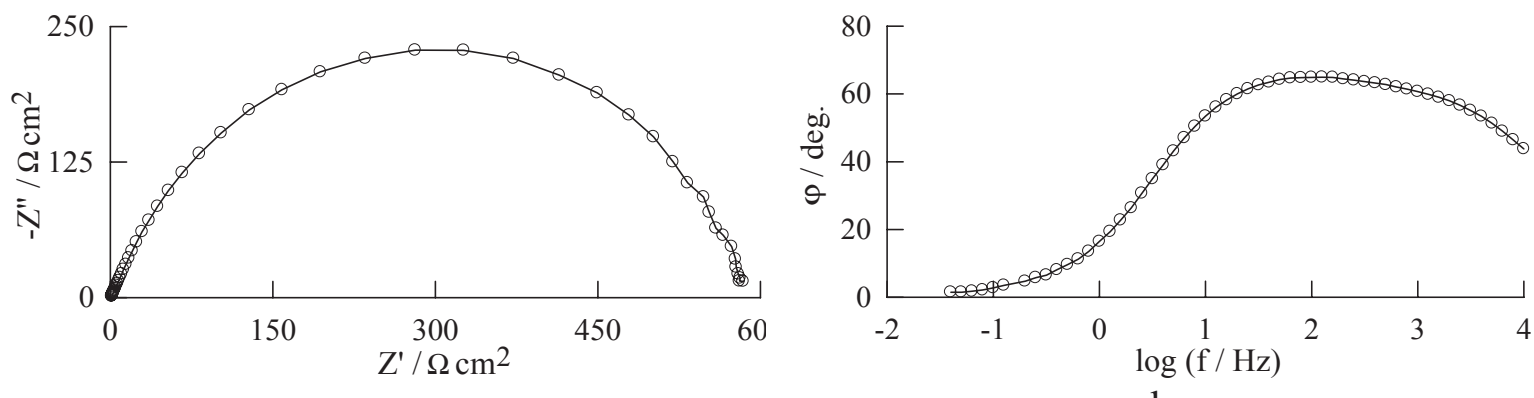

a
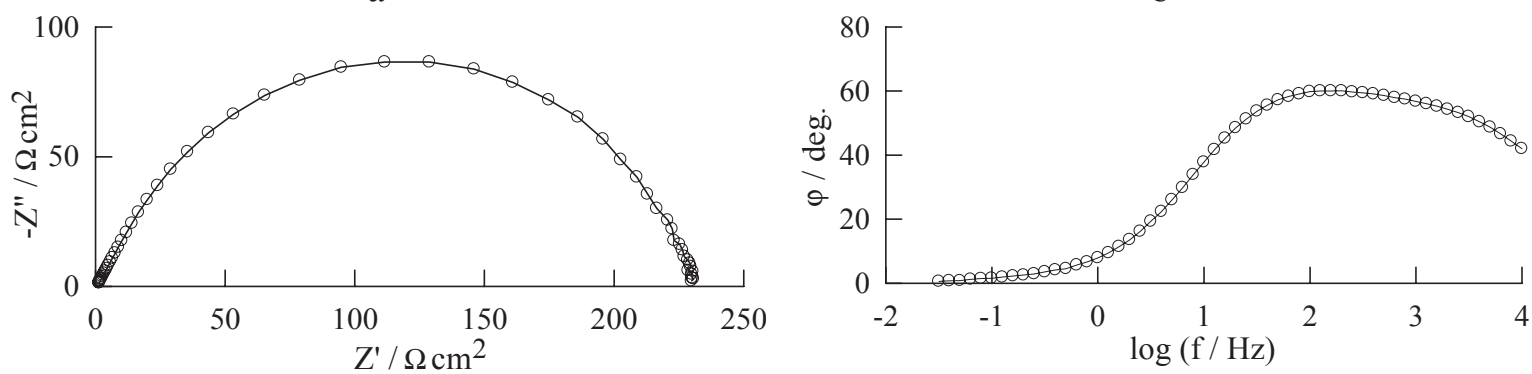

$\mathrm{c}$

d
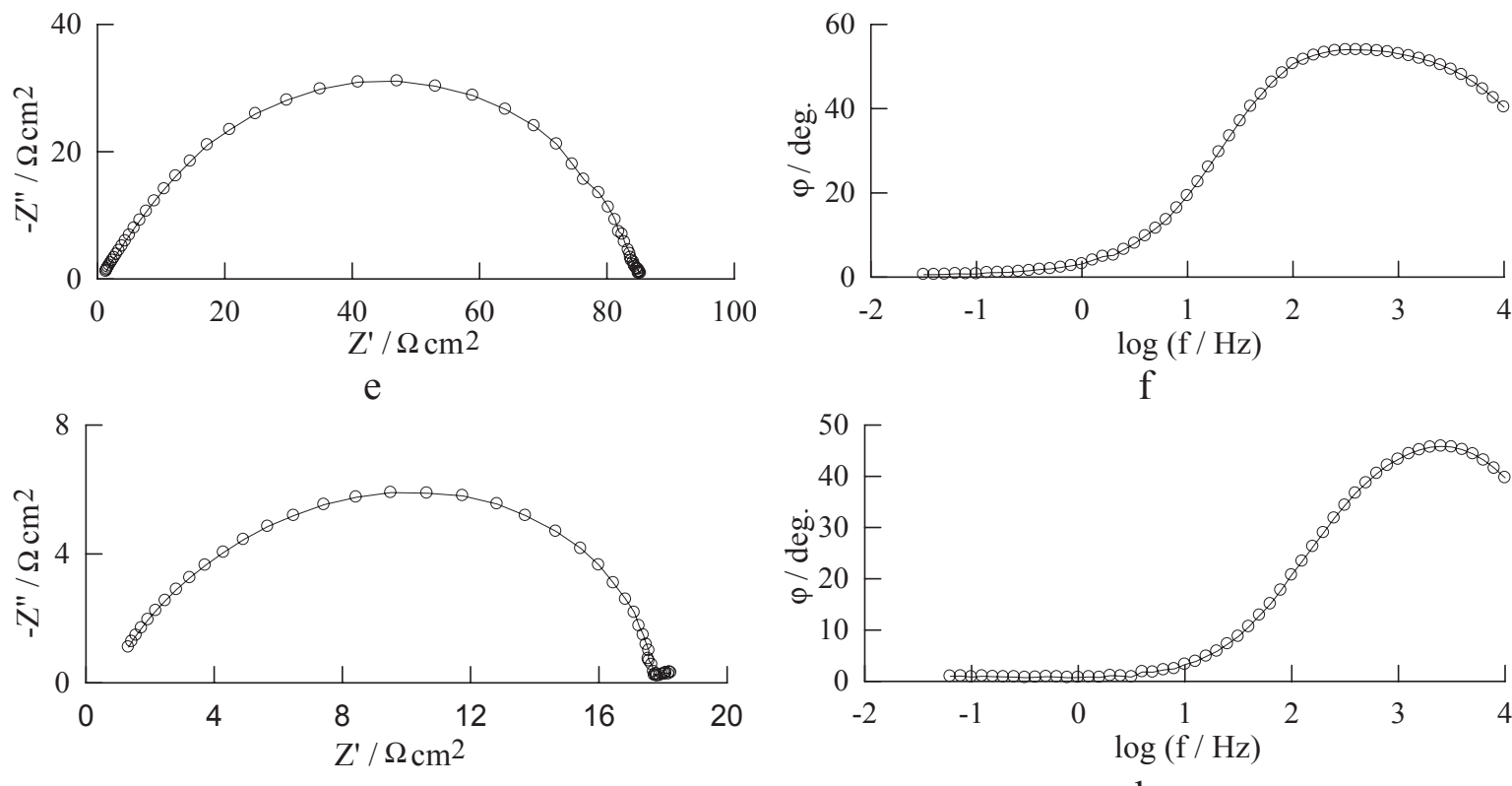

$\mathrm{g}$
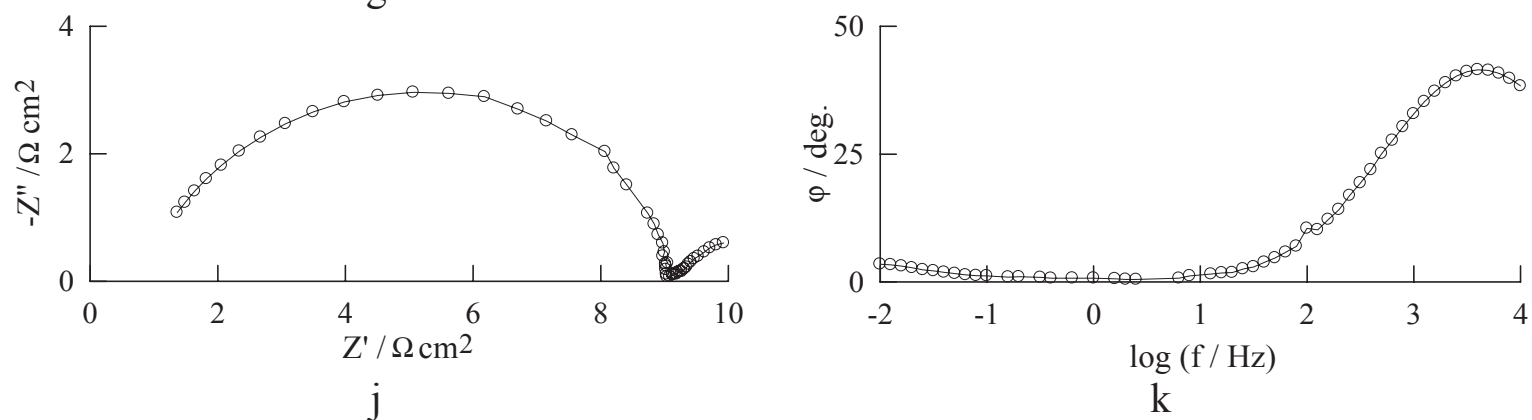

Рис. 3. Графики Найквиста и Боде для NiSi-электрода в $0.5 \mathrm{M} \mathrm{H}_{2} \mathrm{SO}_{4}$ при $E$, В: $-0.16(a, b) ;-0.2(c, d) ;-0.24(e, f) ;-0.32(g, h) ;-0.38(j, k)$

[Fig. 3. Nyquist and Bode plots for NiSi electrode in $0.5 \mathrm{M} \mathrm{H}_{2} \mathrm{SO}_{4}$ at $E, \mathrm{~V}:-0.16(a, b) ;-0.2(c, d) ;-0.24(e, f) ;-0.32(g, h) ;-0.38(j, k)]$ 


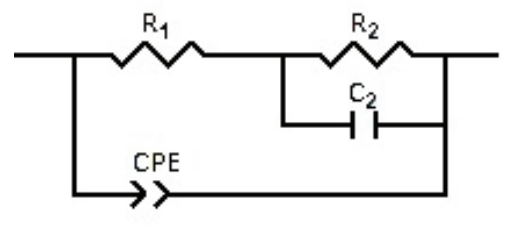

A

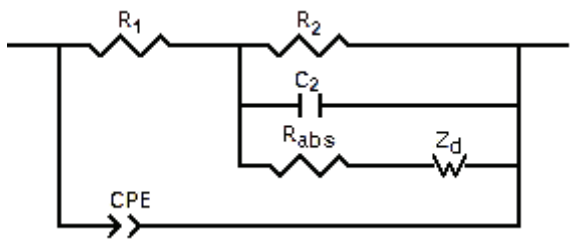

$\mathrm{B}$

Рис. 4. Эквивалентные электрические схемы: А - для РВВ, В - для РВВ + РАВ. Сопротивление раствора не показано

[Fig. 4. Equivalent electrical circuits: A - for HER, B - for HER + HAR. The solution resistance is not shown]

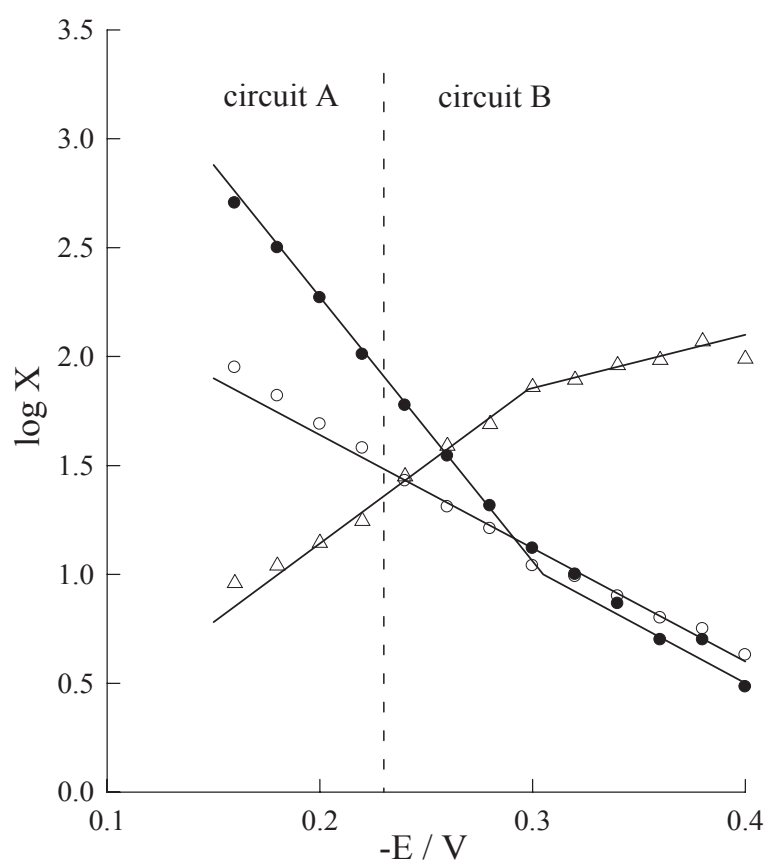

Рис. 5. Зависимости параметров импеданса РBВ от потенциала NiSi-электрода в $0.5 \mathrm{M} \mathrm{H}_{2} \mathrm{SO}_{4}$. ○- $\lg R_{1}$, •$\lg R_{2}, \triangle-\lg C_{2}$. Сопротивления $R_{1}$ и $R_{2}-$ в Ом·см ${ }^{2}$, емкость

$$
C_{2}-\text { в мк } \Phi / \mathrm{cm}^{2}
$$

[Fig. 5. Potential dependences of the HER impedance parameters for NiSi electrode in $0.5 \mathrm{M} \mathrm{H}_{2} \mathrm{SO}_{4}$. $\bigcirc-\log R_{1}$, - $-\log R_{2}, \triangle-\log C_{2}$. The resistances $R_{1}$ and $R_{2}$ are in $\Omega \mathrm{cm}^{2}$, the capacitance $C_{2}$ is in $\mu \mathrm{F} \mathrm{cm}{ }^{-2}$ ]

$E>-0.22$ В и при $E<-0.34$ В наблюдаются отклонения. Малый наклон $d \lg \tau / d E \approx 4 \mathrm{~B}^{-1}$ может наблюдаться в двух случаях $[18,19]$ :

1) РВВ протекает по механизму Фольмера-Тафеля, и замедленной является реакция Фольмера. Малая величина $d \lg \tau / d E$ связана с относительно медленным изменением степени заполнения $\theta$ поверхности электрода адсорбированным водородом с потенциалом электрода для данного механизма [20]: $d \lg \theta / d E=\alpha_{1} F /(2 \cdot 2.3 R T)$, где $\alpha_{1}-$ коэффициент переноса стадии разряда.

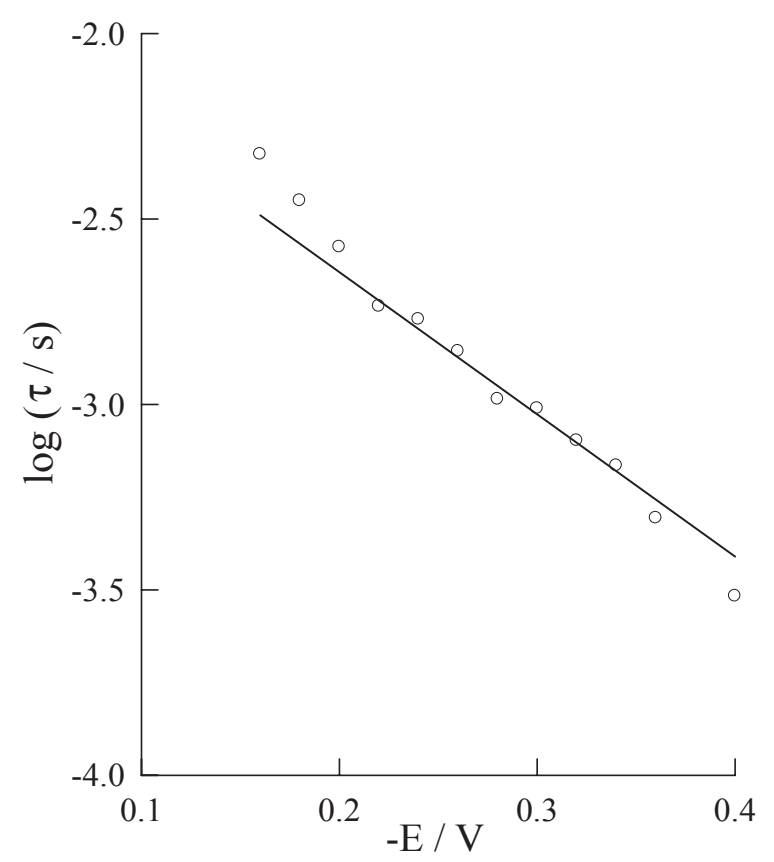

Рис. 6. Зависимость $\lg \tau$ от $E$ для $\mathrm{NiSi}$ в $0.5 \mathrm{M} \mathrm{H}_{2} \mathrm{SO}_{4}$ [Fig. 6. Dependence of $\lg \tau$ on $E$ for NiSi in $0.5 \mathrm{M} \mathrm{H}_{2} \mathrm{SO}_{4}$ ]

2) РВВ протекает по двухмаршрутному механизму Фольмера-Гейровского-Тафеля. В этом случае на зависимости $\lg \tau$ от $E$ имеется прямолинейный участок с наклоном $4.2 \mathrm{~B}^{-1}$ в определенном интервале потенциалов электрода, где выполняются соотношения $k_{3}>k_{2}, k_{3}>k_{1}\left(k_{1}, k_{2}, k_{3}-\right.$ константы скорости стадий Фольмера, Гейровского и Тафеля соответственно) [19].

Учитывая наличие излома на тафелевской зависимости (рис. 1) и отклонений от прямолинейной зависимости в координатах $\lg \tau-E$ (рис. 6), можно предположить, что для $\mathrm{NiSi-электрода} \mathrm{в}$ растворе серной кислоты выполняется второй вариант, то есть выделение водорода на моносилициде никеля происходит по двухмаршрутному механизму. 
Общие выражения $R_{1}, R_{2}$ и $C_{2}$ для двухмаршрутного механизма приведены в [21]. Если учесть еще стадию абсорбции водорода, протекающей с кинетическим контролем, то получим:

$$
\begin{gathered}
R_{1}=-\frac{1}{a_{1}+a_{2}}, \\
R_{2}=-\frac{\left(a_{1}-a_{2}\right)\left(b_{1}+b_{2}\right)}{\left(a_{1}+a_{2}\right)\left[2\left(a_{2} b_{1}-a_{1} b_{2}\right)-\left(a_{1}+a_{2}\right)\left(b_{3}+b_{a}\right)\right]}, \\
C_{2}=q_{1} \frac{\left(a_{1}+a_{2}\right)^{2}}{\left(a_{1}-a_{2}\right)\left(b_{1}+b_{2}\right)},
\end{gathered}
$$

где $a_{k}=\left(\partial i_{k} / \partial E\right)_{\theta}, b_{k}=\left(\partial i_{k} / \partial \theta\right)_{E}, i_{k}-$ скорость $k$-й стадии в единицах плотности тока, индексы 1,2 , $3, a$ относятся к стадиям Фольмера, Гейровского, Тафеля и абсорбции соответственно, $\theta$ - степень заполнения поверхности адсорбированным водородом, $q_{1}$ - заряд, расходуемый на образование монослоя $\mathrm{H}_{\text {алс. }}$ При выводе выражений (1)-(3) катодный ток считали положительным.

Некоторые результаты расчетов временной константы по соотношениям (2) и (3) в предположении изотермы Ленгмюра для адсорбированного водорода приведены на рис. 7. При расчетах принимали, что $k_{3}$ и константа скорости абсорбции водорода $k_{a}$ не зависят от $E$, а для констант скорости реакций Фольмера и Гейровского в прямом и обратном направлениях использовали зависимости $k_{j}=k_{j}^{0} \exp \left(-\alpha_{j} F \eta / R T\right)$ и $k_{-j}^{0} \exp \left(\left(1-\alpha_{j}\right) F \eta / R T\right)$, где $j=1,2, \eta=E-E_{e q}, E_{e q}-$ равновесный потенциал водородного электрода, $k_{j}^{0}$ и $k_{-j}^{0}-$ значения констант скорости при $\eta=0, \alpha_{j}-$ коэффициент переноса. В определенной области потенциалов имеется линейный участок с наклоном $4.2 \mathrm{~B}^{-1}$ (близким к экспериментальному значению $\left.3.8 \mathrm{~B}^{-1}\right)$; протяженность этого участка увеличивается при увеличении константы скорости стадии рекомбинации $k_{3}$. В отсутствие РАВ наклон $d \lg \tau / d E$ при невысоких катодных поляризациях близок к $16 \mathrm{~B}^{-1}$, а при наличии стадии абсорбции водорода (при не слишком больших $k_{a}$ ) $d \lg \tau / d E \approx 7.2 \mathrm{~B}^{-1}$ при $E>-0.2$ В (рис. 7, кривая 3), что хорошо согласуется с экспериментальным значением $d \lg \tau / d E \approx 7 \mathrm{~B}^{-1}$ при $E>-0.22 \mathrm{~B}$ (рис. 6). Эти результаты могут указывать на то, что эквивалентная схема А (рис. 4), которая применима при $E \geq-0.22 \mathrm{~B}$, соответствует в данном случае процессу РВВ + РАВ (абсорбция водорода протекает с кинетическим контролем).

Отличие предполагаемого механизма РВВ на $\mathrm{NiSi}$ в растворе серной кислоты от механизма PBB на FeSi в тех же условиях (одномаршрутный механизм Фольмера-Гейровского) [11] можно связать с тем, что перенапряжение выделения водорода на NiSi значительно ниже (примерно на 0.2 В) по сравнению с $\mathrm{FeSi}$, а также с различием энергии адсорбции водорода на двух силицидах. В области низких перенапряжений возрастает роль стадии рекомбинации в удалении водорода с поверхности электрода [22], что может объяснить появление второго маршрута в механизме PBB на $\mathrm{NiSi-электроде.}$

Таким образом, на основании полученных результатов можно предположить, что адсорбция атомарного водорода на NiSi-электроде в $0.5 \mathrm{M}$ $\mathrm{H}_{2} \mathrm{SO}_{4}$ описывается уравнением изотермы Ленгмюра, выделение водорода происходит по двум параллельным путям - Фольмера-Гейровского и Фольмера-Тафеля, одновременно с РВВ протекает РАВ (с кинетическим контролем при $E>-0.24$ В и с диффузионно-кинетическим контролем при $E \leq-0.24$ B).

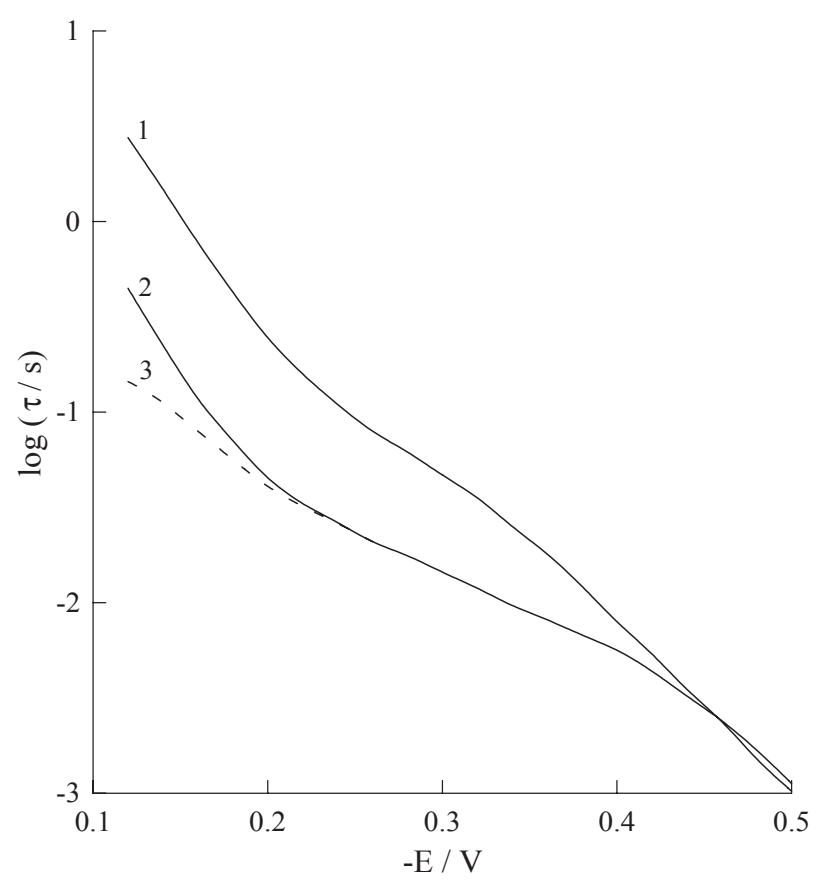

Рис. 7. Расчетные зависимости $\lg \tau$ от $E$ для двухмаршрутного механизма РВВ с учетом РАВ при $k_{1}^{0}=1 \cdot 10^{-10}$, $k_{-1}^{\mathrm{o}}=1 \cdot 10^{-6}, k_{2}^{\mathrm{o}}=1 \cdot 10^{-11}, \alpha_{1}=\alpha_{2}=0.5$. Кривая $1-k_{3}=1 \cdot 10^{-8}$; кривые $2,3-k_{3}=1 \cdot 10^{-7}$; кривые $1,2-k_{a}=0$; кривая 3 $k_{a}=1 \cdot 10^{-8}$. Все константы скорости - в моль $/\left(\mathrm{cm}^{2} \cdot \mathrm{c}\right)$

[Fig. 7. Calculated dependences of $\lg \tau$ on $E$ for two-route HER mechanism with HAR at $k_{1}^{0}=1 \cdot 10^{-10}, k_{-1}^{0}=1 \cdot 10^{-6}$, $k_{2}^{0}=1 \cdot 10^{-11}, \alpha_{1}=\alpha_{2}=0.5$. Curve $1-k_{3}=1 \cdot 10^{-8}$; curves $2,3-k_{3}=1 \cdot 10^{-7}$; curves $1,2-k_{a}=0$; curve $3-k_{a}=1 \cdot 10^{-8}$. All the rate constants are expressed in $\mathrm{mol} \mathrm{cm} \mathrm{cm}^{-2} \mathrm{~s}^{-1}$ ] 


\section{Раствор гидроксида калия}

Поляризационная кривая NiSi-электрода в растворе 1 М КОН представляет собой тафелевскую зависимость с наклоном 0.13 В (рис. 8). Среднеквадратическая погрешность среднего значения потенциала при данной плотности тока составляла 5 мВ. Плотность тока обмена $i_{0}=4.2 \cdot 10^{-6} \mathrm{~A} / \mathrm{cm}^{2}$, то есть близка к $i_{0}$ в кислом растворе. Перенапряжение выделения водорода на NiSi в $1 \mathrm{M} \mathrm{KOH} \mathrm{на} 0.1$ B ниже, чем на FeSi в тех же условиях [11].

В щелочном растворе дифференциальная емкость NiSi-электрода имеет обычные для твердых металлических материалов значения (рис. 9), которые превышают значения $C$ для FeSi-электрода [11]. Характер изменения дифференциальной емкости с потенциалом электрода такой же, как в кислом растворе (рис. 2).

Примеры графиков импеданса для $\mathrm{NiSi-элек-}$ трода в 1 М КОН приведены на рис. 10. Графики Найквиста представляют собой несколько искаженные полуокружности в емкостной полуплоскости, графики Боде при невысоких поляризациях представляют собой несимметричные пики, а при повышении поляризации они становятся более симметричными. Спектры импеданса удовлетворительно описываются с помощью эквивалентной схемы А (рис. 4). Относительные ошибки определения элементов эквивалентной схемы составляли: для $R_{1}-4-7 \%$, для $R_{2}-2-5 \%$ и для $C_{2}-2.5-10.5 \%$ (до $23 \%$ при наиболее низких потенциалах).

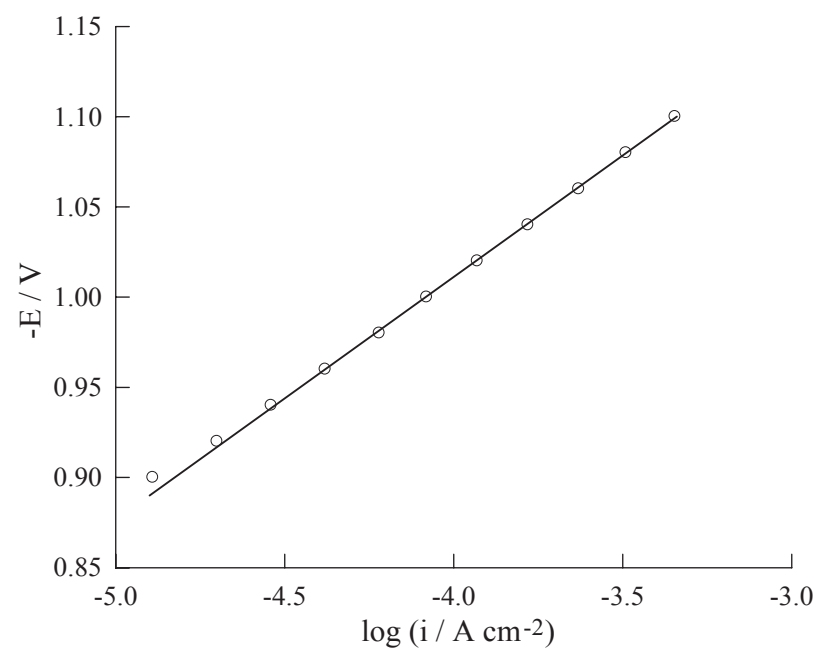

Рис. 8. Поляризационная кривая NiSi-электрода в $1 \mathrm{M} \mathrm{KOH}$

[Fig. 8. Polarization curve for NiSi electrode in $1 \mathrm{M} \mathrm{KOH}]$
Зависимости параметров импеданса РBВ на $\mathrm{NiSi} \mathrm{в} \mathrm{щелочном} \mathrm{растворе} \mathrm{приведены} \mathrm{на} \mathrm{рис.} 11$. Для NiSi в $1 \mathrm{M} \mathrm{KOH} d \lg \tau / d E \geq 7 \mathrm{~B}^{-1}$, то есть примерно в 2 раза больше, чем в растворе $\mathrm{H}_{2} \mathrm{SO}_{4}$ при средних катодных поляризациях.

Качественно результаты импедансных измерений для NiSi в растворе КОН близки к результатам для $\mathrm{Co}_{2} \mathrm{Si}$ в тех же условиях [10]. Соответственно, полученным для NiSi результатам можно дать такую же интерпретацию, как для $\mathrm{Co}_{2} \mathrm{Si}$ : атомарный водород на поверхности $\mathrm{NiSi}$ в щелочном растворе подчиняется изотерме адсорбции Ленгмюра, выделение водорода происходит по механизму Фольмера-Гейровского при неравных коэффициентах переноса стадий, при $E<-1.05$ В обе стадии являются полностью необратимыми.

\section{ВЫВОДЫ}

1. В растворе $0.5 \mathrm{M} \mathrm{H}_{2} \mathrm{SO}_{4}$ адсорбция атомарного водорода на NiSi-электроде описывается уравнением изотермы Ленгмюра, выделение $\mathrm{H}_{2}$ происходит по двухмаршрутному механизму ФольмераГейровского-Тафеля, одновременно протекает абсорбция водорода (с кинетическим контролем при $E>-0.24$ В и с диффузионно-кинетическим контролем при $E \leq-0.24 \mathrm{~B})$.

2. В растворе $1 \mathrm{M} \mathrm{KОН} \mathrm{адсорбция} \mathrm{атомарного}$ водорода на $\mathrm{NiSi-электроде} \mathrm{описывается} \mathrm{уравне-}$ нием изотермы Ленгмюра, выделение $\mathrm{H}_{2}$ происхо-

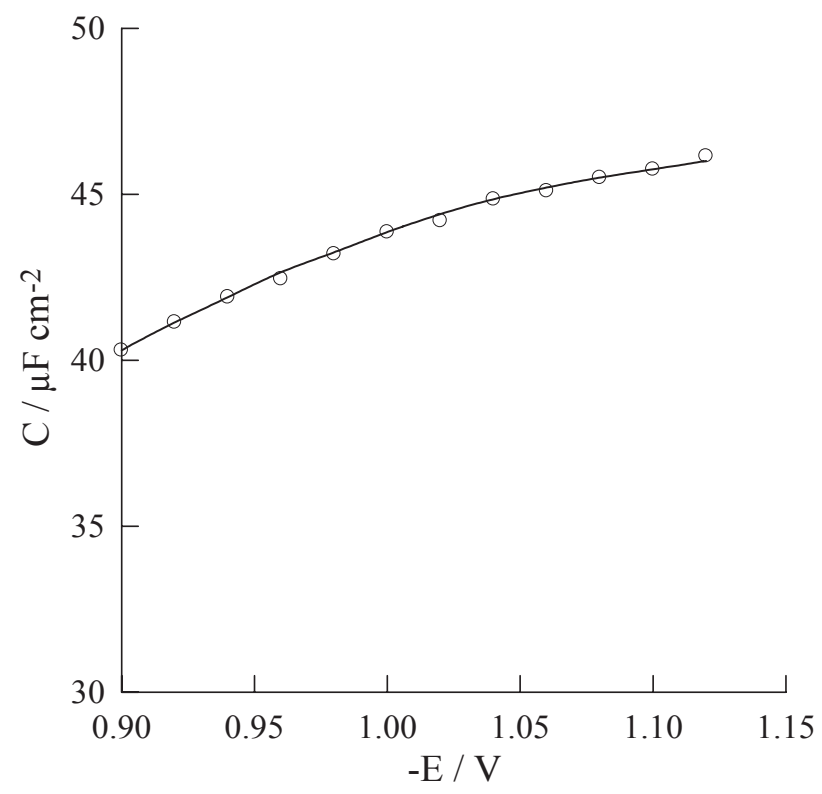

Рис. 9. Кривая дифференциальной емкости при $f=10$ кГц для NiSi-электрода в $1 \mathrm{M} \mathrm{KOH}$

[Fig. 9. Differential capacitance at $f=10 \mathrm{kHz}$ for NiSi electrode in $1 \mathrm{M} \mathrm{KOH}]$ 

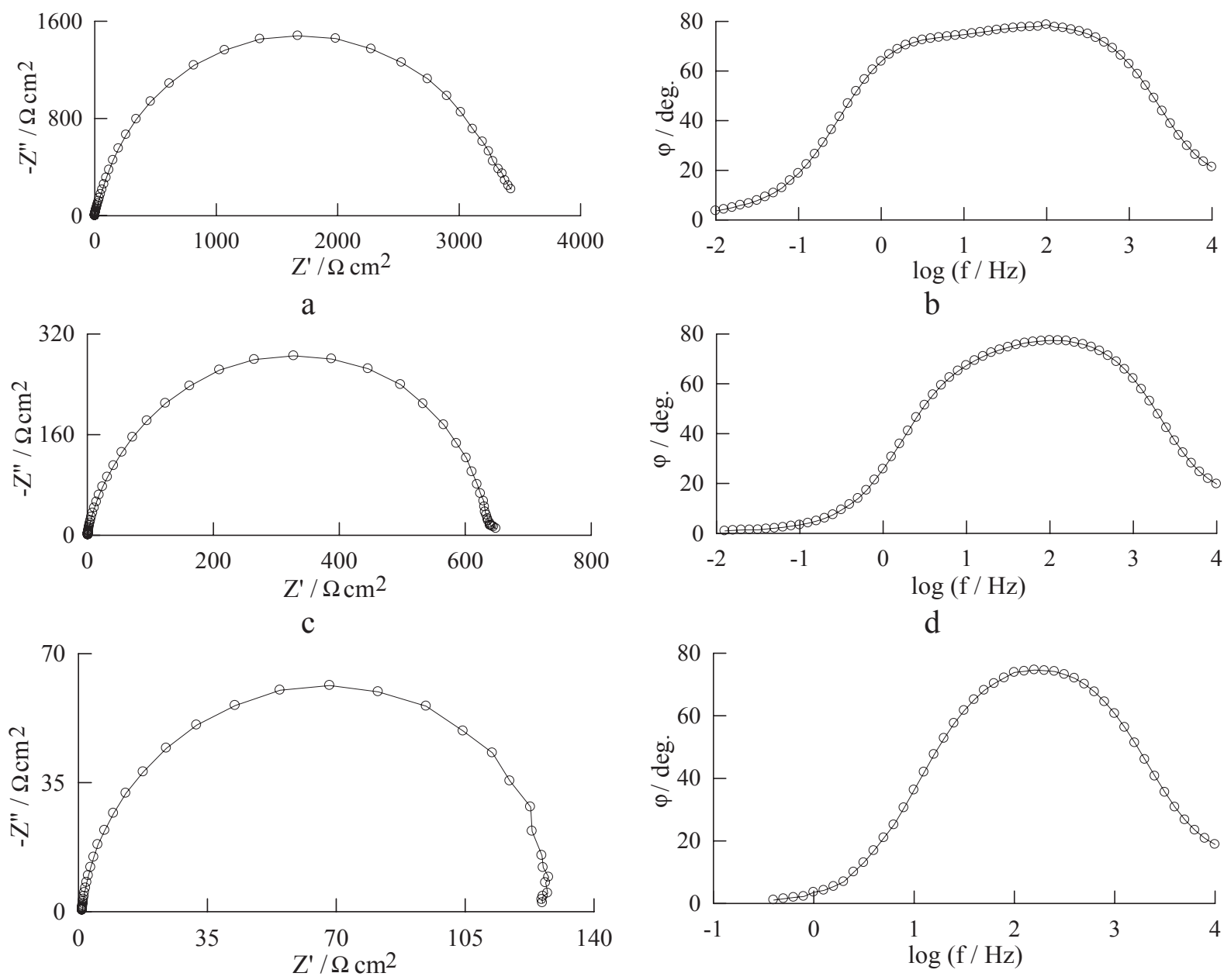

$\mathrm{f}$

Рис. 10. Графики Найквиста и Боде для NiSi-электрода в 1 М КОН при $E$, B: $-0.9(a, b) ;-1.0(c, d) ;-1.1(e, f)$

[Fig. 10. Nyquist and Bode plots for NiSi electrode in $1 \mathrm{M} \mathrm{KOH}$ at $E$, V: $-0.9(a, b) ;-1.0(c, d) ;-1.1(e, f)$ ]

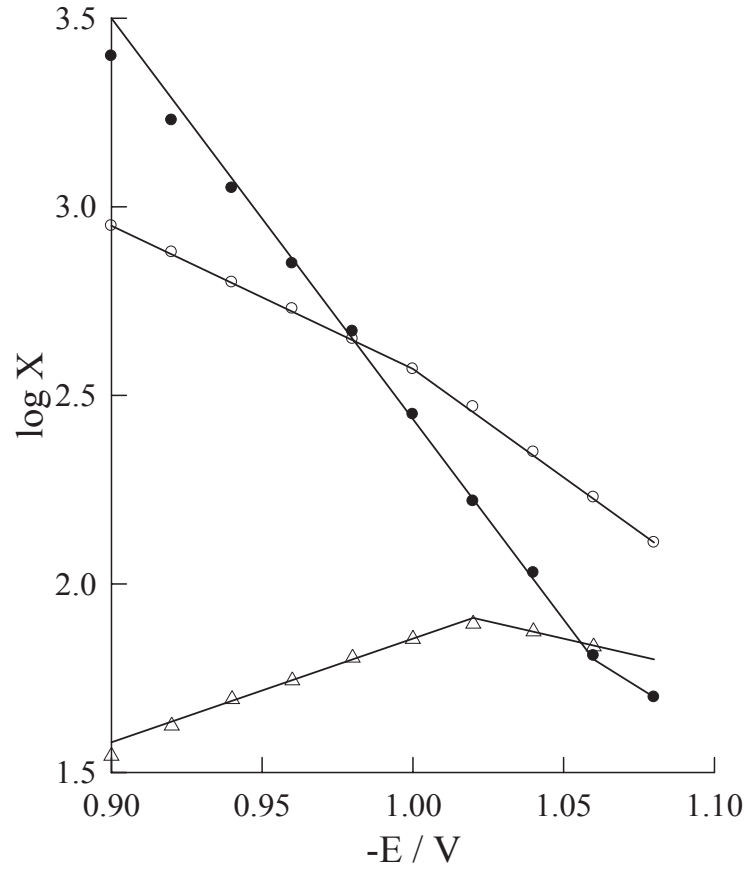

Рис. 11. Зависимости параметров импеданса РВВ от потенциала NiSi-электрода в $1 \mathrm{M} \mathrm{KOH.} \circ-\lg R_{1}, \bullet-\lg R_{2}$, $\Delta-\lg C_{2}$. Сопротивления $R_{1}$ и $R_{2}-$ в Ом.см ${ }^{2}$, емкость $C_{2}$ - В МК $\Phi / \mathrm{cM}^{2}$

[Fig. 11. Potential dependences of the HER impedance parameters for NiSi electrode in $1 \mathrm{M} \mathrm{KOH} . \circ-\log R_{1}, \bullet$ $\log R_{2}, \Delta-\log C_{2}$. The resistances $R_{1}$ and $R_{2}$ are in $\Omega \mathrm{cm}^{2}$, the capacitance $C_{2}$ is in $\mu \mathrm{F} \mathrm{cm}^{-2}$ ] 
дит по механизму Фольмера-Гейровского, $\alpha_{1} \neq \alpha_{2}$; при $E<-1.05$ В обе стадии являются полностью необратимыми.

\section{СПИСОК ЛИТЕРАТУРЫ}

1. Введенский А. В., Гуторов И. А., Морозова Н. Б. // Конденсированные среды и межфазнье границь, 2010, т. 12, № 3, с. 288-300.

2. Safizadeh F., Ghali E., Houlachi G. // Int. J. Hydrogen Energy, 2015, vol. 40, № 1, pp. 256-274.

3. Sapountzi F. M., Gracia J. M., Weststrate C. J., Fredriksson H. O. A., Niemantsverdriet J. W. // Progress in Energy and Combustion Science, 2017, vol. 58, pp. 135 .

4. Shamsul Huq A. K. M., Rosenberg A. J. // J. Electrochem. Soc., 1964, vol. 111, № 3, pp. 270-278.

5. Tilak B. V., Ramamurthy A. C., Conway B. E. // Proc. Indian Acad. Sci. (Chem. Sci.), 1986, vol. 97, № 3-4, pp. 359-393.

6. Vijh A.K., Bélanger G., Jacques R. // Materials Chemistry and Physics, 1989, vol. 21, p. 529-538.

7. Vijh A. K., Bélanger G., Jacques R. // Int. J. Hydrogen Energy, 1990, vol. 15, № 11, pp. 789-794.

8. Vijh A. K., Bélanger G. // J. Mater. Sci. Lett., 1995, vol. 14, pp. 982-984.

9. Поврозник В. С., Шеин А. Б. // Защчита металлов, 2007, т. 43, № 2, c. 216-221.
10. Kichigin V. I., Shein A. B. // Electrochim. Acta, 2015, vol. 164, pp. 260-266.

11. Кичигин В. И., Шеин А. Б., Шамсутдинов А. Ш. // Конденсированные среды и межфазные границы, 2016, т. 18 , № 3, с. 326-337.

12. Pomoni K., Krontiras Ch. // J. Phys. D: Appl. Phys., 1988, vol. 21, pp. 780-783.

13. Valeri S., Del Pennino U., Sassaroli P. // Surface Science, 1983, vol. 134, pp. L537-L542.

14. Harrington D. A., Conway B. E. // Electrochim. Acta, 1987, vol. 32, № 12, pp. 1703-1712.

15. Lasia A. Electrochemical Impedance Spectroscopy and its Applications. New York, Springer, 2014. 367 p.

16. Cordoba-Torres P., Mesquita T. J., Nogueira R. P. // J. Phys. Chem. C, 2015, vol. 119, № 8, pp. 4136-4147.

17. Lim C., Pyun S.-I. // Electrochim. Acta, 1993, vol. 38, № 18, pp. 2645-2652.

18. Kichigin V. I., Shein A. B. // Electrochim. Acta, 2014, vol. 138, pp. 325-333.

19. Kichigin V. I., Shein A. B. // Electrochim. Acta, 2016, vol. 201, pp. 233-239.

20. Devanathan M. A. V., Stachurski Z. // J. Electrochem. Soc., 1964, vol. 111, № 5, pp. 619-623.

21. Кичигин В. И., Шерстобитова И. Н., Кузнецов В. В. // Электрохимия, 1976, т. 12, № 2, с. 315-318.

22. Krishtalik L. I. Adv. Electrochem. Electrochem. Engng, vol. 7 / Ed. by P. Delahay. New York, Intersci. Publ., 1970, p. 283-340.

\title{
THE KINETICS OF THE HYDROGEN EVOLUTION REACTION ON NICKEL MONOSILICIDE IN ACIDIC AND ALKALINE SOLUTIONS
}

\author{
(C) 2017 V. I. Kichigin, A. B. Shein, A. Sh. Shamsutdinov \\ Perm State University, 15 Bukirev str., 614990 Perm, Russia \\ e-mail: ashein@psu.ru; kichigin@psu.ru
}

Received 22.02.2017

\begin{abstract}
The kinetics of the hydrogen evolution reaction on nickel silicide NiSi (fabricated by Czochralski method) in solutions of $0.5 \mathrm{M} \mathrm{H}_{2} \mathrm{SO}_{4}$ and $1 \mathrm{M} \mathrm{KOH}$ has been studied by polarization measurements and electrochemical impedance spectroscopy. In an acid solution two Tafel slopes have been observed on the polarization curve for NiSi: the slope $b$ is equal to $0.105 \mathrm{~V} /$ decade at $E>-0.33 \mathrm{~V}$ (SHE) and $b=0.18 \mathrm{~V} /$ decade at $E<-0.33 \mathrm{~V}$. In an alkaline solution one linear region of the Tafel line has been observed, $b=0.13 \mathrm{~V} /$ decade. The exchange current density $i_{0}$ is $4.45 \cdot 10^{-6} \mathrm{~A} \mathrm{~cm}^{-2}$ and $4.2 \cdot 10^{-6} \mathrm{~A} \mathrm{~cm}^{-2}$ in acidic and alkaline solution respectively. In an acidic solution the Nyquist plots consist of a single semicircle at not high cathodic polarizations and of two well-resolved capacitive arcs at higher cathodic polarizations. An equivalent circuit considering the hydrogen absorption reaction was used at high cathodic polarizations. In an alkaline solution the Nyquist plots consist of a single capacitive semicircle. The potential dependences of equivalent circuit elements $R_{1}, R_{2}, C_{2}$ and time constant $R_{2} C_{2}$ have been discussed. The conclusion was made that hydrogen evolution on NiSi in a sulfuric acid solution proceeds via a two-route Volmer-Heyrovsky-Tafel mechanism (with hydrogen absorption reaction in parallel) and in an alkaline solution via the Volmer-Heyrovsky mechanism with unequal transfer coefficients for the steps. The obtained results show that the adsorbed atomic hydrogen on an NiSi electrode obeys the Langmuir isotherm in both solutions.
\end{abstract}

Keywords: nickel monosilicide, hydrogen evolution reaction, hydrogen absorption, impedance. 


\section{КИНЕТИКА РЕАКЦИИ ВЫДЕЛЕНИЯ ВОДОРОДА НА МОНОСИЛИЦИДЕ НИКЕЛЯ...}

\section{REFERENCES}

1. Vvedenski A. V., Gutorov I. A., Morozova N. B. Condensed Matter and Interfaces, 2010, vol. 12, no. 3, pp. 288-300. Available at: http://www.kcmf.vsu.ru/resources/t_12_3_2010_012.pdf (in Russian)

2. Safizadeh F., Ghali E., Houlachi G. Int. J. Hydrogen Energy, 2015, vol. 40, no. 1, pp. 256-274. DOI: 10.1016/ j.ijhydene.2014.10.109

3. Sapountzi F. M., Gracia J. M., Weststrate C. J., Fredriksson H. O. A., Niemantsverdriet J. W. Progress in Energy and Combustion Science, 2017, vol. 58, pp. 1-35. DOI: $10.1016 /$ j.pecs.2016.09.001

4. Shamsul Huq A. K. M., Rosenberg A. J. J. Electrochem. Soc., 1964, vol. 111, no. 3, pp. 270-278. DOI: 10.1149/1.2426107

5. Tilak B. V., Ramamurthy A. C., Conway B. E. Proc. Indian Acad. Sci. (Chem. Sci.), 1986, vol. 97, no. 3-4, pp. 359-393. DOI: 10.1007/BF02849200

6. Vijh A. K., Bélanger G., Jacques R. Materials Chemistry and Physics, 1989, vol. 21, pp. 529-538. DOI: 10.1016/0254-0584(89)90151-X

7. Vijh A. K., Bélanger G., Jacques R. Int. J. Hydrogen Energy, 1990, vol. 15, no. 11, pp. 789-794. DOI:10.1016/03603199(90)90014-P

8. Vijh A. K., Bélanger G. J. Mater. Sci. Lett., 1995, vol.14, pp. 982-984. DOI: 10.1007/bf00274625

9. Povroznik V. S., Shein A. B. Protection of Metals, 2007, vol. 43, no. 2, pp. 203-207. DOI: 10.1134/ S0033173207020130

10. Kichigin V. I., Shein A. B. Electrochim. Acta, 2015, vol. 164, pp. 260-266. DOI: 10.1016/j.electacta. 2015.02.198

11. Kichigin V. I., Shein A. B., Shamsutdinov A. Sh. Condensed Matter and Interfaces, 2016, vol. 18, no. 3, pp. 326-337. Available at: http://www.kcmf.vsu.ru/resources/t_18 3 2016_003.pdf (in Russian)

12. Pomoni K., Krontiras Ch. J. Phys. D: Appl. Phys., 1988, vol. 21, pp. 780-783. DOI: 10.1088/0022-3727/ $21 / 5 / 017$

13. Valeri S., Del Pennino U., Sassaroli P. Surface Science, 1983, vol. 134, no. 3, pp. L537-L542. DOI: 10.1016/0039-6028(83)90056-0

14. Harrington D. A., Conway B. E. Electrochim. Acta, 1987, vol. 32, no. 12, pp. 1703-1712. DOI: 10.1016/00134686(87)80005-1

15. Lasia A. Electrochemical Impedance Spectroscopy and its Applications. Springer, New York, 2014.

16. Cyrdoba-Torres P., Mesquita T. J., Nogueira R. P. J. Phys. Chem. C, 2015, vol. 119, no. 8, pp. 4136-4147. DOI: $10.1021 /$ jp512063f

17. Lim C., Pyun S.-I. Electrochim. Acta, 1993, vol. 38, no. 18, pp. 2645-2652. DOI: 10.1016/0013-4686(93) 85082-A

18. Kichigin V. I., Shein A. B. Electrochim. Acta, 2014, vol. 138, pp. 325-333. DOI: 10.1016/j.electacta.2014. 06.114

19. Kichigin V. I., Shein A. B. Electrochim. Acta, 2016, vol. 201, pp. 233-239. DOI: 10.1016/j.electacta.2016. 03.194

20. Devanathan M. A. V., Stachurski Z. J. Electrochem. Soc., 1964, vol. 111, no. 5, pp. 619-623. DOI: $10.1149 / 1.2426195$

21. Kichigin V. I., Sherstobitova I. N., Kuznetsov V. V. Electrochemistry, 1976, vol. 12, no. 2, pp. 315-318. (in Russian)

22. Krishtalik L. I. In: P. Delahay (Ed.), Adv. Electrochem. Electrochem. Engng. Vol.7, New York, Intersci. Publ., 1970, pp. 283-340.
Кичигин Владимир Иванович - к. х. н., доцент кафедры физической химии химического факультета Пермского государственного национального исследовательского университета; тел.: +7(342) 2396452, e-mail: kichigin@psu.ru

Шеин Анатолий Борисович - д. х. н., профессор, зав. кафедрой физической химии химического факультета Пермского государственного национального исследовательского университета; тел.: +7(342) 2396468, e-mail: ashein@psu.ru

Шамсутдинов Артем Шамилевич - магистрант кафедры физической химии химического факультета Пермского государственного национального исследовательского университета; тел.: +7(342) 2396452
Kichigin Vladimir I. - Dr. Sci. (Chem.), Associate Professor, Department of Physical Chemistry, Perm State University, ph.: +7(342) 2396452, e-mail: kichigin@psu. $\mathrm{ru}$

Shein Anatoliy B. - Dr. Sci. (Chem.), Professor, Head of Physical Chemistry Department, Perm State University; ph.: +7(342) 2396468, e-mail: ashein@psu.ru

Shamsutdinov Artem Sh. - graduate student, Physical Chemistry Department, Perm State University; ph.: +7(342) 2396452 